Canadian

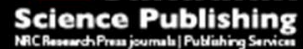

Canadian Journal of Microbiology Revue canadienne de de microbiologie

\title{
Common Dyes Used to Determine Bacterial Polysaccharides on Agar Are Affected by Medium Acidification
}

\begin{tabular}{|r|l|}
\hline Journal: & Canadian Journal of Microbiology \\
\hline Manuscript ID & cjm-2016-0743.R1 \\
\hline Manuscript Type: & Note \\
\hline Date Submitted by the Author: & 06-Feb-2017 \\
\hline Complete List of Authors: & $\begin{array}{l}\text { Hawkins, Justin P.; University of Manitoba, Microbiology } \\
\text { Geddes, Barney A.; University of Manitoba, Microbiology } \\
\text { Oresnik, Ivan John; University of Manitoba, Microbiology }\end{array}$ \\
\hline Keyword: & Sinorhizobium, pH, Aniline blue, Congo red, Calcofluor white \\
\hline
\end{tabular}

SCHOLARONE ${ }^{\text {IM }}$

Manuscripts 
Common Dyes Used to Determine Bacterial Polysaccharides on Agar Are Affected by Medium Acidification

By: Justin P. Hawkins, Barney A. Geddes, and Ivan J. Oresnik

Corresponding author:

Ivan Oresnik

Dept. of Microbiology,

University of Manitoba

Winnipeg, MB

R3T 2N2

CANADA

Tel: $\quad 204-474-7587$

Fax: 204-474-7603

Email: Ivan.Oresnik@umanitoba.ca 


\begin{abstract}
In this work we highlight effects of $\mathrm{pH}$ on bacterial phenotypes when using the bacteriological dyes Aniline blue, Congo red, and Calcofluor white to analyze polysaccharide production. Study of galactose catabolism in Sinorhizobium meliloti led to the isolation of a mutation in $d g o k 1$, which was observed to overproduce exopolysaccharides when grown in the presence of galactose. When this mutant strain was spotted onto plates containing Aniline blue, Congo red, or Calcofluor white, the intensity of the associated staining was strikingly different when compared to the Wild-type. Additionally, a Calcofluor dull phenotype was observed, suggesting production of a polysaccharide other than succinoglycan. Further investigation of this phenotype revealed that these results were dependent on medium acidification, as buffering at pH 6 had no effect on these phenotypes, while medium buffered at $\mathrm{pH} 6.5$ resulted in a reversal of the phenotypes. Screening for mutants of the dgokl strain that were negative for the aniline blue phenotype yielded a strain carrying a mutation in $t k t 2$ which is annotated as a putative transketolase. Consistent with the plate phenotypes, when this mutant was grown in broth cultures it did not acidify its growth medium. Overall this work shows that caution should be exercised in evaluating polysaccharide phenotypes based strictly on the use of dyes.
\end{abstract}

Key Words: Sinorhizobium, pH, Aniline blue, Congo red, Calcofluor white 
Bacteriological dyes have been used as an important component in medium to determine production of various polysaccharides and extracellular elements. In particular, Congo red, Calcofluor white, and Aniline blue have seen prominent use in bacteria, plants, and fungi. Congo red and Calcofluor white are typically used as dyes to stain polysaccharides and cellular elements containing $\beta(1-3)$ or $\beta(1-4)$ linkages, and have particularly strong interactions with cellulose and chitin (Hughes and McCully 1975; Kneen and Larue 1983; Wood 1983; Mori and Bellani 1996; Rivera et al. 2012). However, the use of these dyes is not limited to polysaccharides since Congo red can also bind to extracellular fibers called Curli produced by Escherchia coli (Reichhardt et al. 2016). The use of these dyes has also had an impact on the ability to isolate symbiotically effective rhizobia. Most Rhizobium strains lack the ability to bind Congo red when grown on YEM (Zevenhuizen et al. 1986). However, non-nodulating strains of Rhizobium leguminosarum bv. trifollii have been isolated on the basis of binding to Congo red. This has resulted in the use of Congo red as a potential indicator of nodulating strains of R.leguminosarum bv. trifollii in mixed cultures (Bromfield and Jones 1980). Calcofluor white is used as an indicator for production of the polysaccharide succinoglycan (EPS-I) in S. meliloti Rm1021, and it has long been used as a screening tool to isolate mutants which affect production of this symbiotically important polysaccharide (Leigh et al. 1985, 1987; Long et al. 1988).

The dye Aniline blue is largely utilized to detect the production of callose, a linear $\beta(1-3)$ glucan found in cell walls in both plants and fungi (Stone et al. 1984). This is visualized through a bright yellow fluorescent phenotype when observed under UV light. Fluorescence is due to the interaction of a fluorochrome from Aniline blue (Sirofluor) interacting with $\beta(1-3)$ linkages (Evans et al. 1984). While bacteria do not produce callose, production of similar polysaccharides consisting of $\beta(1-3)$ linked glucans has been observed, including the polysaccharide curdlan 
(Kenyon and Buller 2002; Stone 2005). Production of curdlan is known to occur in various bacteria including some Agrobacterium species (Stasinopoulos et al. 1999). The production of curdlan is visualized in media supplemented with Aniline blue by cell biomass turning a blue colour (Stasinopoulos et al. 1999).

The production of polysaccharides can be heavily influenced by many factors such as $\mathrm{pH}$, salt concentration, osmolarity, and the ability to metabolize carbon sources (Dylan et al. 1990; Miller-Williams et al. 2006; Hellweg et al. 2009; Geddes et al. 2014). Previous work to identify S. meliloti mutants unable to catabolize galactose led to the isolation of a Tn5 insertion mutant in dgoK1 (SRmD304), which was observed to acidify medium when grown in the presence of galactose (Geddes and Oresnik 2012a, 2012b; Geddes et al. 2014). When SRmD304 was initially streaked on Rhizobium Minimal Medium (RMM) (Broughton et al. 1986) using galactose and glycerol as carbon sources, a mucoid phenotype was observed suggesting an increased production of EPS. However, when the strain was streaked onto RMM containing Calcofluor it exhibited a dull fluorescent phenotype that seemed to be atypical for EPS-I (Figure 1c, top panel).

To further investigate the produced polysaccharide, SRmD304 was screened on medium supplemented with either Aniline blue, Congo red, or Calcofluor white. Rm1021 (Wild-type) and SRmD304 were grown at $30^{\circ} \mathrm{C}$ overnight in $5 \mathrm{~mL}$ cultures of LB broth (Sambrook et al. 1989) to an $\mathrm{OD}_{600}$ of 1.0. A $1 \mathrm{~mL}$ aliquot of each culture was pelleted and re-suspended in $100 \mu \mathrm{L}$ of $0.85 \% \mathrm{w} / \mathrm{v}$ saline. Finally, $20 \mu \mathrm{L}$ of this cell suspension was spotted onto YMA (Yeast Mannitol Agar) agar (Kneen and Larue 1983) supplemented with $15 \mathrm{mM}$ galactose and Congo red (25 $\mu \mathrm{g} /$ $\mathrm{mL})$, and RMM supplemented with $15 \mathrm{mM}$ galactose and either Aniline blue $(50 \mu \mathrm{g} / \mathrm{mL})$ or Calcofluor white $(0.02 \%)$. 
The results showed that after 5 days at $30^{\circ} \mathrm{C} \mathrm{Rm} 1021$ had a negative reaction on plates supplemented with either Congo red or Aniline blue (Light orange and white respectively) (Figure 1, top panels). However, a Calcofluor bright phenotype was observed suggesting only succinoglycan was being produced, which is consistent with previous results (Zevenhuizen et al., 1986; Leigh and Lee, 1988). SRmD304 gave a positive result with both Aniline blue and Congo red, turning blue and red respectively, while displaying a dull fluorescence under UV on plates containing Calcofluor white. A possible interpretation of this result was that in the presence of galactose SRmD304 may be producing a novel EPS that contained $\beta(1-3)$ linkages; possibly curdlan. The operon $\operatorname{crdASC}$ encodes enzymes involved in the biosynthesis of curdlan in Agrobacterium (Stasinopoulos et al. 1999), however orthologues to these genes are not found in S. meliloti Rm1021 genome.

Further characterization of SRmD304 showed that, when the dgoK1 strain was grown in weakly buffered conditions in the presence of galactose and a secondary carbon source that it could metabolize it would acidify its growth medium to $\mathrm{pH} 4.5$, and that the EPS that was produced had a 7:1 glucose:galactose ratio that was indicative of succinoglycan (Geddes et al. 2014).

Many dyes are known to be affected by media $\mathrm{pH}$, including Congo red, Aniline blue, and Calcofluor (Darken 1962; Evans et al. 1984; Mera and Davies 1984). To determine if the observed dye phenotypes associated with $d g o K 1$ resulted from medium acidification, the mutant and the wild-type strains were spotted onto plates that were modified by adding $50 \mathrm{mM}$ MOPS buffered at either $\mathrm{pH} 6$ or $\mathrm{pH}$ 6.5. The results show that when the wild-type (Rm1021) and the strain carrying the $d g o K 1$ mutation (SRmD304) were spotted onto plates buffered at $\mathrm{pH} 6.5$ the phenotypes that were noted for SRmD304 on the weakly buffered plates differed. The intensity 
of the staining with Congo red and Aniline blue decreased, whereas the fluorescence associated with Calcofluor white increased (Figure 1, middle panels). In contrast, when the strains were spotted onto plates buffered at $\mathrm{pH} 6$ the phenotypes of both the wild-type and the strain carrying the $d g o K 1$ mutation appeared similar to that seen when SRmD304 was spotted onto the weakly buffered media, resulting in a positive reaction to Aniline blue and Congo red while being Calcofluor dull (Figure 1, bottom panels). Taken together these observations support the hypothesis that the dye phenotypes associated with the $d g o K 1$ mutation were due to medium acidification.

The influence of $\mathrm{pH}$ on Aniline blue phenotypes has not to our knowledge been previously shown at this $\mathrm{pH}$ range. It was reasoned that if the positive reaction of Aniline blue is correlated with medium acidification in S. meliloti, this property could be exploited to screen for mutant strains unable to acidify their growth medium. To test this hypothesis, SRmD338, which carries an unmarked deletion of $d g o k 1$ (Geddes 2014), was mutagenized with Tn5 as previously described (Finan et al. 1988). The resulting Tn5 mutants were screened on RMM supplemented with $15 \mathrm{mM}$ galactose and Aniline blue. Mutants that were phenotypically white were purified, and the site of insertion in the $S$. meliloti genome was determined using arbitrary PCR (MillerWilliams et al. 2006). Two such mutants were isolated in this manner; one in the gene $S M c 00588$, which has been previously shown to be necessary for medium acidification in a dgok1 mutant background (Geddes et al. 2014), and one in the gene $t k t 2$, encoding for a putative transketolase. The $t k t 2:: \operatorname{Tn} 5$ mutation was transduced into Rm1021 to further investigate phenotypes associated with this mutation. It was determined that a strain carrying this mutation had a carbon utilization phenotype similar to those reported for transketolase mutants in E. coli (Zhao and Winkler 1994). The growth phenotypes were fully complemented by the introduction 
of a plasmid over-expressing tkt2 (Data to be presented elsewhere).

To determine if the Aniline blue phenotype associated with SRmD373 ( $\Delta d g o K 1$, $t k t 2:: \operatorname{Tn} 5)$ was due to an inability to acidify its growth medium, broth cultures of SRmD373 were grown in RMM medium containing both glycerol and galactose as previously described (Geddes 2014). Consistent with what was previously observed, the wild-type (Rm1021) did not acidify its growth medium whereas supernatant of the SRmD338 ( $\Delta$ dgoK1) growth medium dropped to a $\mathrm{pH}$ around 4.5 (Figure 2). In contrast, SRmD373 ( $\Delta d g o K 1$, tkt2::Tn5) was markedly impaired in its ability to acidify its growth medium (Figure 2).

The isolation of a mutation in $t k t 2$ suggests alteration of central carbon metabolism plays a role in the observed galactose dependent medium acidification observed in SRmD338. This could be due to altered carbon flow to compounds which are secreted and subsequently drop the $\mathrm{pH}$ of the medium. Medium acidification in the $d g o k l$ background has been shown to be dependent on galactose dehydrogenase activity (SMc00588), suggesting that galactonate accumulation plays a role in altering the medium (Geddes and Oresnik 2012b). The observation that a mutation in $t k t 2$ results in a similar phenotype suggests that medium acidification may not be solely dependent upon SMc00588. We have also observed that a strain carrying only a $t k t 2$ mutation has an Aniline blue negative phenotype (white) under conditions which Rm1021 stains blue (Data to be presented elsewhere). This suggests a mutation in $t k t 2$ may affect medium acidification independent of the $d g o k 1$ mutation and galactose. Since the loss of transketolase represents a major block in central metabolism, it seems plausible that altered carbon flow to other metabolites may also affect medium acidification. Of note, it has been recently shown that proteins associated with the pentose phosphate pathway are increased when $S$. meliloti is grown in acidic conditions (Draghi et al. 2016). 
The results observed here show previously un-reported dye phenotypes of Aniline blue, Calcofluor white and Congo red. While it is well known that these dyes have $\mathrm{pH}$ components, it is usually at extreme $\mathrm{pH}$ ranges, and have not been reported around $\mathrm{pH} 6-\mathrm{pH}$ 6.5. Although our results suggest that the attributes associated with Aniline blue can be exploited to screen for phenotypes, it is important to emphasize that these results suggest caution should be used when using these dyes to assign polysaccharide phenotypes if they are being used in weakly buffered media.

\section{Acknowledgements}

This work was funded by a Natural Sciences and Engineering Research Council of Canada (NSERC) Discovery grant awarded to IJO. JPH acknowledges support from the University of Manitoba Faculty of Science Award and the University of Manitoba Faculty of Graduate Studies GETS program. BAG acknowledges funding in the form of an NSERC CGS-D award.

\section{References}

Bromfield, E.S.P. and Jones, D.G. (1980) A strain marker in Rhizobium trifolii based on the absorption of congo-red. Zentrabil Bakteriol Naturwiss 135: 290-295.

Broughton, W.J., Wong, C.H., Lewin, A., Samrey, U., Myint, H., Meyerz, H., et al. (1986) Identification of Rhizobium plasmid sequences involved in recognition of Psophocarpus, Vigna, and other legumes. J Cell Biol 102: 1173-1182.

Darken, M.A. (1962) Absorption and transport of fluorescent brighteners by microorganisms. Appl. Microbiol. 10: 387-393.

Draghi, W.O., Del Papa, M.F., Hellweg, C., Watt, S.A., Watt, T.F., Barsch, A., et al. (2016) A consolidated analysis of the physiologic and molecular responses induced under acid stress 
in the legume-symbiont model-soil bacterium Sinorhizobium meliloti. Sci. Rep. 6: e29278.

Dylan, T., Nagpal, P., Helinski, D.R., and Ditta, G.S. (1990) Symbiotic pseudorevertants of Rhizobium meliloti ndv mutants. J. Bacteriol. 172: 1409-1417.

Evans, N.A., Hoyne, P.A., and Stone, B.A. (1984) Characteristics and specificity of the interaction of a fluorochrome from aniline blue (Sirofluor) with polysaccharides. Carbohydr. Polym. 4: 215-230.

Finan, T.M., Oresnik, I.J., and Bottacin, A. (1988) Mutants of Rhizobium meliloti defective in succinate metabolism. J. Bacteriol. 170: 3396-3403.

Geddes, B.A. (2014) Characterization of genetic loci for carbon metabolism and competition for nodule occupancy in Sinorhizobium meliloti. Phd thesis, University of Manitoba, Winnipeg, Manitoba, Canada

Geddes, B.A., González, J.E., and Oresnik, I.J. (2014) Exopolysaccharide production in response to medium acidification is correlated with an increase in competition for nodule occupancy. Mol. Plant. Microbe. Interact. 27: 1307-1317.

Geddes, B.A. and Oresnik, I.J. (2012a) Genetic characterization of a complex locus necessary for the transport and catabolism of erythritol, adonitol and L-arabitol in Sinorhizobium meliloti. Microbiol. (United Kingdom) 158: 2180-2191.

Geddes, B.A. and Oresnik, I.J. (2012b) Inability to catabolize galactose leads to increased ability to compete for nodule occupancy in Sinorhizobium meliloti. J. Bacteriol. 194: 5044-5053.

Hellweg, C., Pühler, A., and Weidner, S. (2009) The time course of the transcriptomic response of Sinorhizobium meliloti 1021 following a shift to acidic pH. BMC Microbiol. 9: 37. 
Hughes, J. and McCully, M.E. (1975) The use of an optical brightener in the study of plant structure. Stain Technol. 50: 319-29.

Kenyon, W.J. and Buller, C.S. (2002) Structural analysis of the curdlan-like exopolysaccharide produced by Cellulomonas flavigena KU. J Ind Microbiol Biotechnol 29: 200-203.

Kneen, B.E. and Larue, T.A. (1983) Congo red absorption by Rhizobium leguminosarum. Appl. Environ. Microbiol. 45: 1-4.

Leigh, J.A. and Lee, C.C. (1988) Characterization of polysaccharides of Rhizobium meliloti exo mutants that form ineffective nodules. J. Bacteriol. 170: 3327-3332.

Leigh, J.A., Reed, J.W., Hanks, J.F., Hirsch, A.M., and Walker, G.C. (1987) Rhizobium meliloti mutants that fail to succinylate their calcofluor-binding exopolysaccharide are defective in nodule invasion. Cell 51: 579-587.

Leigh, J.A., Signer, E.R., and Walker, G.C. (1985) Exopolysaccharide-deficient mutants of Rhizobium meliloti that form ineffective nodules. Proc. Natl. Acad. Sci. U. S. A. 82: 62316235.

Long, S.R., Reed, J.W., Himawan, J., and Walker, G.C. (1988) Genetic analysis of a cluster of genes required for synthesis of the calcofluor-binding exopolysaccharide of Rhizobium meliloti. J. Bacteriol. 170: 4239-4248.

Mera, S.L. and Davies, J.D. (1984) Differential Congo red staining: the effects of $\mathrm{pH}$, nonaqueous solvents and the substrate. Histochem. J. 16: 195-210.

Miller-Williams, M., Loewen, P.C., and Oresnik, I.J. (2006) Isolation of salt-sensitive mutants of Sinorhizobium meliloti strain Rm1021. Microbiology 152: 2049-2059. 
Mori, B. and Bellani, L.M. (1996) Differential staining for cellulosic and modified plant cell walls. Biotech. Histochem. 71: 71-72.

Reichhardt, C., McCrate, O.A., Zhou, X., Lee, J., Thongsomboon, W., and Cegelski, L. (2016) Influence of the amyloid dye Congo red on curli, cellulose, and the extracellular matrix in E. coli during growth and matrix purification. Anal. Bioanal. Chem. 1-9.

Rivera, L., Jiménez-Zurdo, J.I., Rivas, R., Dazzo, F., Velázquez, E., Martínez-Molina, E., et al. (2012) Role of Rhizobium endoglucanase CelC2 in cellulose biosynthesis and biofilm formation on plant roots and abiotic surfaces. Microb. Cell Fact. 11: 1-12-2859-11-125.

Sambrook, J., Fritsch, E.F., and Mantiatis, T.L. (1989) Molecular cloning: a laboratory manual 2nd ed. Cold Spring Harbor Laboratory Press, Cold Spring Harbor.

Stasinopoulos, S.J., Fisher, P.R., Stone, B.A., and Stanisich, V.A. (1999) Detection of two loci involved in $(1 \rightarrow 3)$ - $\beta$-glucan (curdlan) biosynthesis by Agrobacterium sp. ATCC31749, and comparative sequence analysis of the putative curdlan synthase gene. Glycobiology 9: 3141.

Stone, B. (2005) Callose and related glucans. Encycl. Life Sci. 1-15 doi: 10.1038/npg.els.0004111.

Stone, B.A., Evans, N.A., Bonig, I., and Clarke, A.E. (1984) The application of Sirofluor, a chemically defined fluorochrome from aniline blue for the histochemical detection of callose. Protoplasma 122: 191-195.

Wood, J. (1983) A basis for specific detection and histochemistry of polysaccharides. $J$. Histochem. Cytochem. 31: 823-826. 
Zevenhuizen, L.P.T.M., Bertocchi, C., and Van Neerven, A.R.W. (1986) Congo red absorption and cellulose synthesis by Rhizobiaceae. Antonie Van Leeuwenhoek 52: 381-386.

Zhao, G. and Winkler, M.E. (1994) An Escherichia coli K-12 tktA tktB mutant deficient in transketolase activity requires pyridoxine (vitamin B6) as well as the aromatic amino acids and vitamins for growth. J. Bacteriol. 176: 6134-6138.

\section{Figure legends}

Figure 1. Dye phenotypes in buffered media. Observed dye phenotypes of strains on plates supplemented with A) Congo red B) Aniline blue, and C) Calcofluor white. Bacterial spots were scored after 5 days on media that was buffered as labeled. Calcofluor white images were observed during exposure to UV light.

Figure 2. Measurement of culture $\mathrm{pH}$ for each strain over 72 hours. The data presented are an average of 3 independent biological replicates. Where not visible error bars are smaller than the size of data points. 
Figure 1
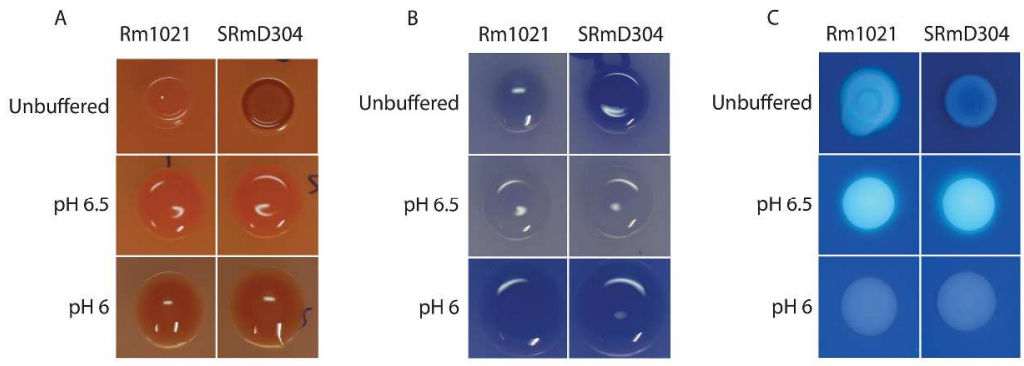

Figure 1. Dye phenotypes in buffered media. Observed dye phenotypes of strains on plates supplemented with A) Congo red B) Aniline blue, and C) Calcofluor white. Bacterial spots were scored after 5 days on media that was buffered as labeled. Calcofluor white images were observed during exposure to UV light.

$279 \times 361 \mathrm{~mm}(300 \times 300 \mathrm{DPI})$ 
Figure 2

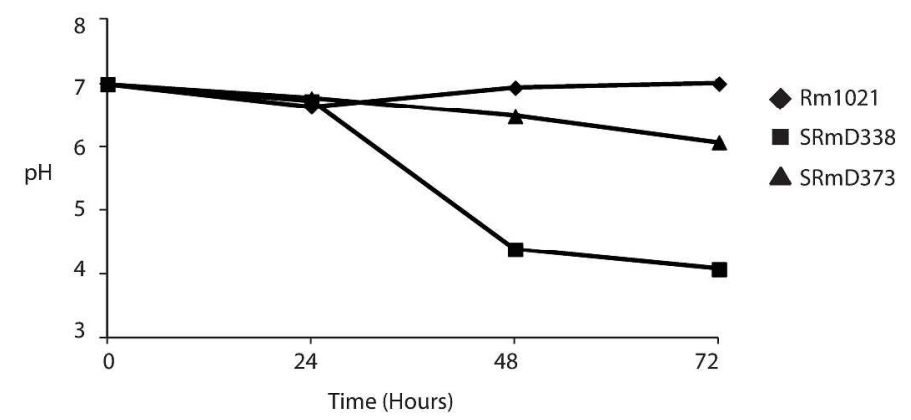

Figure 2. Measurement of culture pH for each strain over 72 hours. The data presented are an average of 3 independent biological replicates. Where not visible error bars are smaller than the size of data points.

$279 \times 361 \mathrm{~mm}(300 \times 300 \mathrm{DPI})$ 